\title{
Properties of Fuzzy Resolving Set
}

\section{Mary Jiny a and R. Shanmugapriya ${ }^{b}$}

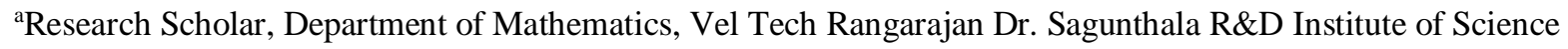
and Technology, Avadi, Chennai, Tamil Nadu, India. E-mail: maryjinymaths@ gmail.com

${ }^{\mathrm{b}}$ Associate Professor, Department of Mathematics, Vel Tech Rangarajan Dr. Sagunthala R\&D Institute of Science and Technology, Avadi, Chennai, Tamil Nadu, India. E-mail: spriyasathish11@gmail.com

Article History: Received: 11 January 2021; Accepted: 27 February 2021; Published online: 5 April 2021

Asbract: In a fuzzy graph $G(v, \sigma, \mu)$, for a subset $H$ of $\sigma$, the representation of $\sigma-H$ with respect to $H$ in terms of strength of connectedness of vertices are distinct then $H$ is called the fuzzy resolving set of $G$. In this article, we discuss the properties of fuzzy resolving set and fuzzy resolving number. And also proved some theorems and properties of fuzzy resolving number in fuzzy cyclic and fuzzy labeling graphs.

Keywords: Fuzzy Labeling Graph, Fuzzy Cycle, Resolving Set, Fuzzy Resolving Set, Fuzzy Resolving Number.

\section{Introduction}

Lotfi Asker Zadeh explained the Fuzzy Mathematics in 1965, later in the year 1975, Fuzzy Graph theory is introduced by Rosenfield. The fuzzy graph is a current research area and it has plenty of applications in real life. Therefore a lot of researchers do research in fuzzy graph. There are different types of fuzzy graphs namely fuzzy labeliing graphs, fuzzy cyclic graphs, intustiastic fuzzy graph, complete fuzzy graphs, irregular fuzzy graphs, regular fuzzy graphs etc.. The concept of colouring, labeling, domination, fuzzy clique etc., are the research areas which are more demanding. Slater in 1975, explained the concept of Resolving number of a graph. It is applied to find the position of the robot differently in a graph-structured framework. If we assume that the robots are moving in a fuzzy graph-structured framework and it needs to identify its position uniquely in terms of safety level, this motivated Shanmugapriya and Jiny to introduced the fuzzy resolving set, fuzzy resolving number, fuzzy super resolving set and a fuzzy super resolving number in the year 2019. As an extension of the research work, we will discuss some properties of resolving number of a fuzzy graph in this paper.

\section{Definitions}

Definition 2.1 Let $V$ be a non-empty set of vertices, $\sigma$ is the function from set of all vertices $V$ to $[0,1]$ and $\mu$ is a function from $V \times V$ to $[0,1]$ such that for all $a, b \in V, \mu(a, b) \leq \sigma(a) \wedge \sigma(b)$, then the ordered triple $G(V, \sigma, \mu)$ is called the fuzzy graph. The support of $\mu$ and $\sigma$ are given as $\mu^{*}=\{(u, v) / \mu(a, b)>0\}$ and $\sigma^{*}=$ $\{u / \sigma(u)>0\} .[2]$.

Definition 2.2 Strength (or weight) of the path $P=u_{1}, u_{2}, \ldots u_{n}$ is the membership value of the weakest edge in the path $P$. The path $P$ is called a cycle if $u_{1}=u_{n}$.

Definition 2.3 The weight (or strength)of connectedness between the two vertices $u_{1}$ to $u_{n}$ is the maximum of strength of all paths between $u_{1}$ to $u_{n}$, is denoted as $\mu^{\infty}\left(u_{1}, u_{n}\right)$ [3], for simplicity of our usage, we use $w\left(u_{1}, u_{n}\right)$.

Definition 2.4 An edge $u v$ is strong if $\mu^{\infty}(u, v)=\mu(u, v)$. And $u$ is called the strong neighbour of $v$. Strong neighbours of a vertex $u=\left\{v \in V / \mu^{\infty}(u, v)=\mu(u, v)\right\}$.

Definition 2.5 A 2-dominating set $D$ is a subset of $V$ such that for all $x \in V-D$ there exist minimum two strong neighbour in $D$. The minimum cardinality of a 2-dominating set is called 2-domination number of the fuzzy graph $G$ and is denoted as $\gamma_{2}(G)$.

Definition 2.6 Let $G(V, \sigma, \mu)$ be a connected fuzzy graph with number of vertices are greater than 2 . Let $V=$ $\left\{v_{1}, v_{2}, \ldots v_{n}\right\}$, a subset $H=\left\{\sigma_{1}, \sigma_{2}, \ldots \sigma_{k}\right\}$ of $\sigma$ with cardinality greater than or equal to 2 is called the fuzzy resolving set of $G$ if the representation of $\sigma-H=\left\{\sigma_{k+1}, \sigma_{k+2}, \ldots \sigma_{n}\right\}$ with respect to $H, \sigma_{i} / H=$ $\left\{w\left(u_{i}, u_{1}\right), w\left(u_{i}, u_{2}\right), \ldots w\left(u_{i}, u_{k}\right)\right\}, i=k+1, k+2, \ldots n$ are distinct $\forall u_{i} \in(\sigma-H)^{*}$. Which are arranged as a row of a matrix of order $R_{n-k \times k}$ called Fuzzy Resolving Matrix of $G$ with respect to $H$ and is denoted as $R_{n-k \times k}$. The cardinality of the minimum resolving set of $G$ is called the resolving number of $G$ denoted as 
$\operatorname{Fr}(G)$. If the representation of $\sigma$ with respect to $H$ are distinct then $H$ is called the fuzzy super resolving set of $G$. The cardinality of the minimum super resolving set is called the fuzzy super resolving number of $G$, denoted as $\operatorname{Sr}(\mathrm{G}) .[1]$.

Definition 2.7 The Connectedness matrix $C_{i j}$ of the fuzzy graph $G$ is defined as $C_{i j}=\mu^{\infty}\left(v_{i}, v_{j}\right)$ for $i \neq j$ and $C_{i j}=0$ if $i=j$.

Result 1: If $H$ is a fuzzy resolving set of a connceted fuzzy labeling graph $G$, then $H$ is a fuzzy super resolving set of $G .[4]$.

Result 2: Every fuzzy labeling cycle $G^{\omega}$ of ' $2 n-1^{\prime}$ vertices such that $G^{*}$ is a cycle, has a fuzzy resolving set of cardinality ' $n-1$ '.

Note 1: through out this paper we consider a fuzzy graph $G$ with $|V| \leq 3$.

Note 2: For any fuzzy graph $G, 2 \leq|H| \leq n-1$.

Note 3: In this paper, we take the fuzzy set $\sigma=\left\{\left(x_{i}, \sigma\left(x_{i}\right)\right\} \quad i=1,2, \ldots n\right.$ as a crisp set $\sigma=$ $\left\{\sigma_{1}, \sigma_{2}, \ldots \sigma_{n}\right\}$ where $\left(x_{1}, \sigma\left(x_{1}\right)\right)=\sigma_{1}$.

\section{Illustration 2.1}

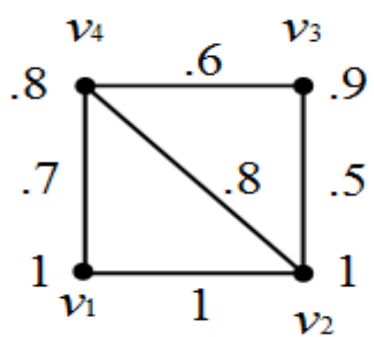

Fig. 1. Fuzzy Graph $G(V, \sigma, \mu)$

$$
\begin{gathered}
V=\left\{v_{1}, v_{2}, v_{3}, v_{4},\right\} ; \sigma=\left\{\left(v_{1}, 1\right),\left(v_{2}, 1\right),\left(v_{3}, .9\right),\left(v_{4}, .8\right)\right\} \\
\mu=\left\{\left(v_{1} v_{2}, 1\right),\left(v_{2} v_{3}, .5\right),\left(v_{3} v_{4}, 6\right),\left(v_{4} v_{1}, .7\right),\left(v_{2} v_{4}, .8\right)\right\} \\
\text { Let } H_{1}=\left\{\sigma_{1}, \sigma_{2}\right\} ; \sigma-H_{1}=\left\{\sigma_{3}, \sigma_{4}\right\} \text { where } \sigma_{1}=\left(v_{1}, \sigma\left(v_{1}\right)\right) \\
\sigma_{3} / H_{1}=\left(w\left(v_{3}, v_{1}\right), w\left(v_{3}, v_{2}\right)\right)=(.6, .6) \\
\sigma_{4} / H_{1}=\left(w\left(v_{4}, v_{1}\right), w\left(v_{4}, v_{2}\right)\right)=(.8, .8)
\end{gathered}
$$

The representation of $\sigma-H_{1}$ with respect to $H_{1}$ are all distinct, therefore $H_{1}$ is the resolving set of $G$. Similarly we can see that, $H_{2}=\left\{\sigma_{1}, \sigma_{3}\right\}, H_{3}=\left\{\sigma_{1}, \sigma_{4}\right\}, H_{4}=\left\{\sigma_{2}, \sigma_{3}\right\}, H_{5}=\left\{\sigma_{2}, \sigma_{4}\right\}$ are all fuzzy resolving set of $G$ and $H_{6}=\left\{\sigma_{3}, \sigma_{4}\right\}$ is not a fuzzy resolving set of $G$. Hence, the minimum resolving set has cardinality 2 , that is, $\operatorname{Fr}(G)=2$.

\section{Properties of a Fuzzy Resolving Set}

Theorem 3.1 For a fuzzy labeling graph $G$ with $n \geq 2$ and $\gamma_{2}(G)=2$, then $\operatorname{Fr}(G)=2=\operatorname{Sr}(G)$.

Proof: Let $G$ be a fuzzy labeling graph. Then there exist a bijection from the set of all edges and vertices to $[0,1]$. If the 2-domination number of $G, \gamma_{2}(G)=2$, then there exist a domination set $D$ with $|D|=2$ such that for all $u \in V-D$, the two elements in $D$ are strong neighbours.

Let $D=\left\{x_{1}, x_{2}\right\}$ then, $\mu^{\infty}\left(u_{i}, x_{1}\right)=\mu\left(u_{i}, x_{1}\right)$ and $\mu^{\infty}\left(u_{i}, x_{2}\right)=\mu\left(u_{i}, x_{2}\right)$ for all $u_{i} \in V-D$.

Now the representation of $\sigma-H$ with respect to $H=\left\{\left(x_{1}, \sigma\left(x_{1}\right)\right),\left(x_{2}, \sigma\left(x_{2}\right)\right)\right\}$;

$\sigma_{i} / H=\left(\mu^{\infty}\left(u_{i}, x_{1}\right), \mu^{\infty}\left(u_{i}, x_{2}\right)\right)=\left(\mu\left(u_{i}, x_{1}\right), \mu\left(u_{i}, x_{2}\right)\right)$ are distinct for all $\sigma_{i} \in V-H$, since $G$ is a fuzzy labeling. Therefore $H$ is a resolving set of $G$ and it is also a fuzzy super resolving set of $G$.

Theorem 3.2 In a fuzzy labeling graph $G$, if there exist a vertex $u$ such that $u v$ is a stron arc for all $v \in$ $G[u \neq v]$ then, $\operatorname{Fr}(G)=\operatorname{Sr}(G)=2$.

Proof: Let $G$ be a fuzzy labeling graph. Then there exist a one-one onto map from the set of all edges and vertices to $[0,1]$. Let $u$ be a vertex in $G$ such that $u v$ is a stron $\operatorname{arc}$ for all $v \in G$ then, $\mu^{\infty}(u, v)=\mu(u, v) \forall v \in$ 
$G$. Now let us take $H=\left\{(u, \sigma(u)),\left(u_{k}, \sigma\left(u_{k}\right)\right)\right\}$ for some $u_{k} \in V\left[u \neq u_{k}\right] . \sigma_{i} / H=\left(\mu^{\infty}\left(u_{i}, u\right), \mu^{\infty}\left(u_{i}, u_{k}\right)\right)=$ $\left(\mu\left(u_{i}, u\right), \mu^{\infty}\left(u_{i}, u_{k}\right)\right)$ which are all distinct since $\mu\left(u_{i}, u\right)$ are all distinct $\left[u_{i} u\right.$ is a strong arc for all $u_{i} \in V$ and $G$ is a fuzzy labeling.]. Therefore $H$ is a fuzzy resolving set and which is also a fuzzy super resolving set by result [1]. Hence $\operatorname{Fr}(G)=\operatorname{Sr}(G)=2$.

Theorem 3.3 Let $G(V, \sigma, \mu)$ be a fuzzy graph with $|V|=4$ and $G^{*}$ is a cycle. If $\mu$ is not a constant function then $\operatorname{Fr}(G)=2$.

Proof: Let $G(V, \sigma, \mu)$ be a fuzzy graph with $|V|=4$ and $G^{*}$ is a cycle. If $\mu$ is not a constant function then there exist at least two distinct edge membership value.

Now let $\mu(u, v)=\vee \mu\left(u_{i}, v_{j}\right) \quad i \neq j, u_{i}, v_{j} \in V$

Choose $H=\left\{(u, \sigma(u)),\left(v_{i}, \sigma\left(v_{i}\right)\right) / v_{i} \neq u\right.$, and $\left.v_{i} \in V\right\}$ or $H=\left\{(v, \sigma(v)),\left(v_{i}, \sigma\left(v_{i}\right)\right) / v_{i} \neq u, v\right.$ and $v_{i} \in$ $V$ \}. Then $H$ is a resolving set of $G$.And hence $\operatorname{Fr}(G)=2$.

Theorem 3.4 Let $G(V, \sigma, \mu)$ be a fuzzy graph with $|V|=5$ and $G^{*}$ is a five cycle. If $\mu$ is not a constant function then the fuzzy resolving number of $G$ is either 2 or 3 .

Proof: Let $G(V, \sigma, \mu)$ be a fuzzy graph with $|V|=5$ and $G^{*}$ is a five cycle. If $\mu$ is not a constant function then there is a posssibility of two, three, four and five distinct edge membership value.

Case(i) If there is exactly two distinct edge weight in $G$.

Then the corresponding edges will be adjacent in at-least one place. Let it be $u v$ and $v w$. choose the non adjacent vertices $u$ and $w$ and then choose any one of the two remaining vertices from $V-\{u, v, w\}$, these three vertices will form the crisp set $H^{*} \in V$ of the resolving set $H \in \sigma$ (the representation of $\sigma-H$ with respect to $H$ will be different). That is there always exist a resolving set of $G$ with cardinality three. If we consider any two element subset $H$ of $\sigma$ whcih will not form a resolving set since there exist only two distinct edge membership value, out of the three representation of $\sigma-H$ with respect to $H$ any two will be the same. And hence from the definition of fuzzy resolving number, the fuzzy resolving number of $G$ in this case is 3 .

Case(ii) If there is exactly three different edge weight in $G$.

Then the three edges with different edge membership value will be consecutive in at least one place of the cycle $G^{*}$ which will form a path. If we have only one such path then choose the two end vertices of the path for $H^{*} \in V$ and the corresponding $H \in \sigma$ will form resolving set of cardinality 2 . And therefore by definition, the fuzzy resolving number of $G$ is 2 .

Case(ii)(a) if we have two such paths and there exist only one weakest edge then choose any one of path, then select the one end vertex of the edge with maximum membership value in the path and choose one end vertex of the edge with minimum membership value which is not adjacent to the chosen vertex for $H^{*} \in V$ and the corresponding $H \in \sigma$ will form resolving set of cardinality 2 .

Case(ii)(b) if we have two such paths and there is two weakest edge (if a cyclic graph $G$ has more than one weakest arc then it is called fuzzy cycle) which are not adjacent then choose the end vertex of the any one path and choose any one of the remaining vertex for $H^{*} \in V$ and the corresponding $H \in \sigma$ will form resolving set of cardinality 3 .

Case(ii)(c) if we have two such paths and there is two weakest edge which are adjacent. Let $\alpha_{1}<\alpha_{2}<\alpha_{3}$ are the three different edge membership value. Now select one vertex of the edge with membership value $\alpha_{2}$ and the vertex of the edge with membership value $\alpha_{3}$ and not adjacent to the selected one for $H^{*} \in V$ and the corresponding $H \in \sigma$ will form resolving set of cardinality 2 .

Case(iii) If there is exactly four different edge weight

Then the corresponding edges will be consecutive in the cycle and which will form a path. then follow the case (ii) to get a resolving set of cardinality 2.

Case (iv) if all five edges have a different edge membership value. 
Then it will form a fuzzy labelling cycle of order 5. then by result [2] the resolving number of $G$ is 2 .

By case (i), (ii),(iii) and (iv) the resolving number of $G$ is either 3 or 2.

Illustration 3.1 The fuzzy resolving number of the fuzzy cycle fig 2(a) is ' 2 ', fig 2(b) is ' 3 ' and fig 2(c) is ' 2 '.

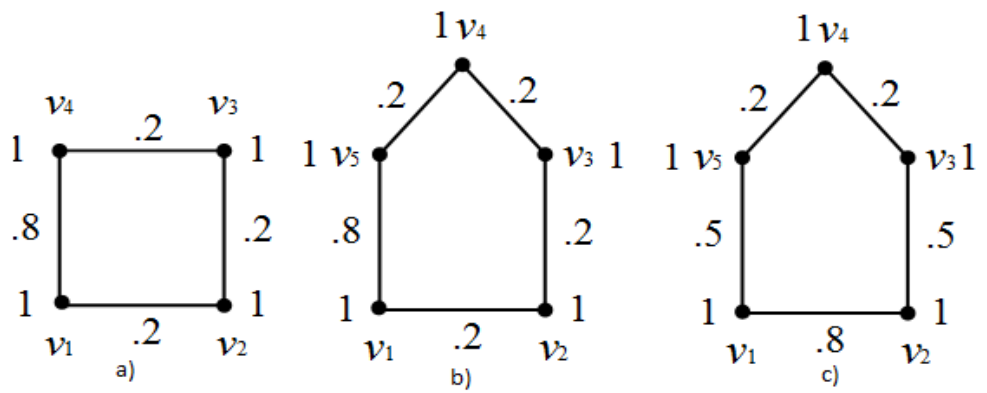

Fig. 2. Cyclic Fuzzy Graphs

Theorem 3.5 Let $G(V, \sigma, \mu)$ be a fuzzy graph with $|V|=n$. If $H$ is a resolving set of $G$ with $|H|=n / 2$ then $\sigma-H$ is does not need to be a resolving set of $G$.

proof: By definition[2.7], the conncectedness matrix of the fuzzy graph $G$ is a symmetric matrix. let $H$ is a subset of $\sigma$ with $|H|=n / 2$. If we put the representation of $\sigma-H$ with respect to $H$ in the row of a $\frac{n}{2} \times \frac{n}{2}$ matrix say $C_{1}$ and the representation of $H$ with respect to $\sigma-H$ in the row of a $\frac{n}{2} \times \frac{n}{2}$ matrix say $C_{2}$. Then $C_{1}$ and $C_{2}$ will be transpose to each other $\left[C_{1}=C_{2}{ }^{T}\right]$. Now let $H$ is the resolving set of $G$ then each row of $C_{1}$ are distinct which implies that each column of $C_{2}$ are distinct. And which does not implies that each row of $C_{2}$ are distinct. Therefore the representation of $H$ with respect to $\sigma-H$ is does not need to be distinct. Hence $\sigma-H$ does not need to be a resolving set of $G$.

Corollary 3.1 In a fuzzy graph $G(V, \sigma, \mu)$, if $H$ is a resolving set of $G$ then $\sigma-H$ is does not need to be a resolving set of $G$.

Illustration 3.2 Let the connectedness matrix of the graph $G$ is

$$
\begin{gathered}
v_{i j}=v_{1} \\
v_{1} \\
v_{3} \\
v_{4}
\end{gathered}\left[\begin{array}{cccc}
0 & v_{2} & v_{3} & v_{4} \\
4 & 0 & .4 & .4 \\
.4 & 0 & .6 & .6 \\
.4 & .6 & 0 & .6 \\
4 & .6 & .6 & 0
\end{array}\right]
$$

Here the subset $H=\left\{\sigma_{3}, \sigma_{4}\right\}$ is a resolving set of $G$.

$$
\begin{aligned}
& \sigma_{1} / H=(.4, .4) \\
& \sigma_{2} / H=(.6, .6)
\end{aligned}
$$

But the subset $\sigma-H=\left\{\sigma_{1}, \sigma_{2}\right\}$ of $\sigma$ is not the resolving set of $G$. Since the representation of $H$ with respect to $\sigma-H$ is not distinct.

$$
\begin{aligned}
& \sigma_{3} / \sigma-H=(.4, .6) \\
& \sigma_{4} / \sigma-H=(.4, .6)
\end{aligned}
$$

\section{Conclusion}

Resolving set of a fuzzy graph is a trending topic in fuzzy graph. In this paper, we have discussed some properties of resolving set of the fuzzy graph. We have proved that the resolving number of fuzzy graph $G$ with $G^{*}$ is a four cycle is 2 and five cycle is 2 or 3 . We have also proved that for a fuzzy labeling graph $G$ if the 2 dominating number is 2 then the resolving number of $G$ is also 2 . We would like to work on finding the relation between the domination set of the fuzzy graph and the resolving set in future. 


\section{References}

1. J.N. Mordeson, S. Mathew, and D.S. Malik, "Fuzzy graph theory with applications to human trafficking" Springer International Publishing, 2018.

2. Nagoor Gani, and Akram, M., "Novel properties of fuzzy labeling graphs", Journal of Mathematics, vol. 1, pp. 1-6, 2014.

3. A.N. Gani, and D.R. Subahashini, "Properties of fuzzy labeling graph", Applied Mathematical Sciences, vol. 6, no. 70, pp. 3461-3466, 2012.

4. D.M. Jiny, and R. Shanmugapriya, "Modified Fuzzy Resolving Number", European Journal of Molecular \& Clinical Medicine, vol. 7, no. 2, pp. 5047-5052, 2020.

5. A.N. Gani, and K.P. Devi, "Bounds on the 2- domination number of a fuzzy graph", International Journal of Pure and Applied Mathematics, Vol. 118, no. 6, pp. 435-441, 2018.

6. J.N. Mordeson, S. Mathew, and D.S. Malik, "Fuzzy graph theory with applications to human trafficking", Springer International Publishing, 2018.

7. M.S. Sunitha, and S. Mathew, "Fuzzy graph theory: a survey", Annals of Pure and Applied mathematics, vol. 4, no. 1, pp. 92-110, 2013. 